\title{
Qualitätssicherung der schmerzmedizinischen Versorgung in Österreich
}

\author{
Klassifikation schmerztherapeutischer Einrichtungen
}

\author{
Wolfgang Jaksch (D) - Rudolf Likar · Erika Folkes · Klaus Machold · Friedrich Herbst · Katharina Pils · Peter Stippl • \\ Sandra Lettner · Mildred Alfons · Richard Crevenna - Christian Wiederer · Janina Dieber · Reinhold Glehr
}

Eingegangen: 31. Januar 2017 / Angenommen: 28. März 2017 / Online publiziert: 19. April 2017

(c) Der/die Autor(en) 2017. Dieser Artikel ist eine Open-Access-Publikation.

Zusammenfassung In Österreich existiert keine flächendeckende Schmerzversorgung, die nur annähernd international geforderten Kriterien erfüllt. Derzeit gibt es etwa 30 interdisziplinäre Schmerzpraxen und -kliniken, die nach einem Konzept der Österreichischen Schmerzgesellschaft (ÖSG) arbeiten, rund 10

Dr. W. Jaksch, MD

Österreichische Schmerzgesellschaft (ÖSG), Wien,

Österreich

\section{Dr. W. Jaksch, MD ( $\varangle)$}

Abteilung für Anästhesie, Intensiv- und

Schmerztherapie, Wilhelminenspital der Stadt Wien,

Montleartstr. 37, 1160 Wien, Österreich

wolfgang.jaksch@chello.at

Prim. Univ.-Prof. Dr. R. Likar · Dr. J. Dieber, MSc Österreichische Gesellschaft für Anästhesiologie, Reanimation und Intensivmedizin (ÖGARI), Wien, Österreich

Prim. Univ.-Prof. Dr. R. Likar

Klinikum Klagenfurt, Klagenfurt, Österreich

Dkfm. E. Folkes

Allianz chronischer Schmerz, Wien, Österreich

Univ.-Prof. Dr. K. Machold

Österreichische Gesellschaft für Rheumatologie und

Rehabilitation (ÖGR), Wien, Österreich

Prim. Univ. Prof. Dr. F. Herbst, FRCS

Österreichische Gesellschaft für Chirurgie (OEGCH), Wien, Österreich

Chirurgische Abteilung, Barmherzige Brüder - Krankenhaus Wien, Wien, Österreich

Prof. Dr. K. Pils

Österreichische Gesellschaft für Geriatrie und Gerontologie (ÖGGG), Wien, Österreich weitere Schmerzambulanzen befinden sich in Bezirksund Landeskrankenhäusern (Stein et al. 2010). Vor wenigen Jahren waren es aber noch an die 50 Schmerzambulanzen. Schließungen von Ambulanzen und Sparmaßnahmen im Gesundheitssektor gefährden jedoch eine adäquate Schmerzversorgung österrei-

Dr. P. Stippl

Österreichischer Berufsverband für Psychotherapie (ÖBVP), Wien, Österreich

Mag. Dr. S. Lettner · Mag. M. Alfons

Berufsverband Österreichischer PsychologInnen (BÖP), Wien, Österreich

ao. Univ.-Prof. Dr. R. Crevenna, MBA MSc .

Prim. Dr. C. Wiederer

Österreichische Gesellschaft für Physikalische Medizin und Rehabilition (ÖGPMR), Wien, Österreich

ao. Univ.-Prof. Dr. R. Crevenna, MBA MSc

Universitätsklinik für Physikalische Medizin, Rehabilitation und Arbeitsmedizin, Wien, Österreich

Prim. Dr. C. Wiederer

Klinikum am Kurpark Baden, Baden, Österreich

Kurhaus Bad Gleichenberg, Bad Gleichenberg, Österreich

Dr. J. Dieber, MSc

Schmerzambulanz, Landeskrankenhaus Hartberg, Hartberg, Österreich

Abt. für Anästhesiologie und Intensivmedizin, Landeskrankenhaus Hartberg, Hartberg, Österreich

Dr. R. Glehr

Österreichische Gesellschaft für Allgemein- und

Familienmedizin (ÖGAM), Wien, Österreich 
chischer Schmerzpatienten. Die Versorgung der rund 1,8 Mio. Österreicher mit chronischen Schmerzen ist durch das österreichweite Fehlen einer Bedarfsplanung schmerzmedizinischer Einrichtungen nicht sichergestellt und vorhandene Strukturen - wie spezialisierte Ambulanzen oder Akutschmerzdienste in Krankenhäusern - basieren in erster Linie auf dem persönlichen Engagement Einzelner. Derzeit werden die unterschiedlichen schmerzmedizinischen Einrichtungen in Österreich mit sehr unterschiedlichen Betriebszeiten geführt, sodass aktuellen Schätzungen des Ist- und Sollbedarfs zufolge $74 \%$ der chronischen Schmerzpatienten keine ambulante Schmerztherapie angeboten wird und etwa 50 vollzeitbetriebene Schmerzambulanzen fehlen.

Unter der Federführung der Österreichischen Schmerzgesellschaft haben verschiedene Fachgesellschaften Struktur und Qualitäts-Kriterien für schmerzmedizinische Einrichtungen in Österreich definiert wie z.B. Nachweis von Fortbildungen, Zusammenarbeit in interdisziplinären Behandlerteams oder Mindestanzahl an neuen Patienten pro Jahr, je nach Klassifizierung der Einrichtung. Dieses Konzept zur abgestuften Versorgung von Schmerzpatienten soll einen ersten Schritt zur Verbesserung der Versorgung von Schmerzpatienten in Östereich darstellen.

Schlüsselwörter Schmerzversorgung · Strukturverbesserung · Qualitätssicherung · Klassifikation · Qualitätskriterien

\section{Quality assurance of pain care in Austria Classification of management facilities}

Summary In Austria there is no nationwide coverage of pain management, which meets even approximately international criteria. At present there are about 30 interdisciplinary pain management offices and clinics providing care according to a concept of the Austrian Pain Society (ÖSG), about 10 other outpatient pain clinics are located in district and country hospitals. A few years ago, there still were about 50 pain clinics. Yet closure of outpatient clinics and cost-cutting measures in the health sector jeopardize adequate pain relief for patients with chronic pain conditions.

Hence, the supply of care for approx. 1.8 mio. Austrians with chronic pain is not guaranteed due to lack of a comprehensive demand planning of pain care facilities. Furthermore, existing structures such as specialized clinics or emergency services in hospitals are primarily based on the personal commitment of individuals. At present, the various centres for pain management in Austria are run with very different operating times, so that for $74 \%$ of the chronic pain patients the desired requirements for outpatient pain management are not met and about 50 full-time pain clinics are missing.
Under the patronage of the Austrian Pain Society, various national specialist societies have defined the structure and quality criteria for pain management centres in Austria, include, among others, proof of training, cooperation in interdisciplinary teams or minimum number of new patients per year, depending on the classification of the institution.

This stepwise concept of care provision for pain patients is intended as first step to help improve the care of pain patients in Austria!

Keywords Pain management - Structural improvement · Quality assurance - Classification - Quality criteria

\section{Einleitung}

Chronische Schmerzen zählen zu den häufigsten Gründen, um Einrichtungen des Gesundheitssystems zu beanspruchen. Sie sind oft für Krankenstände, Frühpensionen und Produktivitätsverluste verantwortlich, haben deutliche Auswirkungen auf die Lebensqualität und stellen darum eine bedeutende Herausforderung für die Gesellschaft und für Public Health dar [26].

Laut Österreichischem Gesundheitsbericht 2014 [25], einer regelmäßig stattfindenden repräsentativen und umfassenden nationalen Befragung zu Gesundheitsstatus, Lebensqualität und sozialen Auswirkungen sowie Inanspruchnahme von Leistungen des Gesundheitssystems, sind derzeit etwa 1,8 Mio. Österreicher $^{1}$ (ca. $25 \%$ ) von chronischen Schmerzen betroffen (länger als 3 Monate), wobei die Prävalenz bei den Frauen in allen Altersklassen höher ist als bei den Männern. Somit liegt Österreich in der Schmerzprävalenz im internationalen Durchschnitt. Jüngere Studien aus europäischen Ländern berichten von einer Schmerzprävalenz zwischen 25 und $35 \%$ [6].

In Österreich existiert keine flächendeckende Versorgung von Patienten mit chronischen Schmerzen, die nur annähernd international anerkannte Kriterien erfüllt: Zur Behandlung des chronischen Schmerzes stehen hierzulande aktuell rund 600 Ärzte (Allgemeinmedizin und Fachärzte) mit einem Zusatzdiplom „Spezielle Schmerztherapie“ der Österreichischen Ärztekammer zur Verfügung. Von 130 Rheumatologen haben lediglich etwa 20 einen Kassenvertrag. Die Österreichische Gesellschaft für Allgemeinmedizin (ÖGAM) bietet ihren Mitgliedern Fort- und Weiterbildung im Rahmen des „Schmerzcurriculums“ an. Zusätzlich werden Schmerzpatienten durch Ärzte verschiedener Fachrichtungen behandelt, die zwar über keine Zusatzaus-

\footnotetext{
${ }^{1}$ Die in dieser Publikation verwendeten Personen- und Berufsbezeichnungen treten der besseren Lesbarkeit halber nur in einer Form auf, sind aber natürlich gleichwertig auf beide Geschlechter bezogen.
} 
bildung, aber langjährige Erfahrung in der Schmerztherapie verfügen [26].

Seitens der Österreichischen Akademie für Psychologie [19] gibt es seit 2008 das Curriculum „Psychologische Schmerzbehandlung“, das sich an Klinische und Gesundheitspsychologen richtet, die in freier Praxis oder im stationären Bereich in der Versorgung von Patienten mit chronischen Schmerzzuständen tätig sind oder in diesem Bereich tätig werden wollen. Zurzeit gibt es in Österreich 24 Klinische und Gesundheitspsychologen, die die Zertifizierung der Zusatzqualifikation für psychologische Schmerzbehandlung erworben haben. Es gibt etwa 30 interdisziplinäre Schmerzpraxen und -kliniken, die nach einem Konzept der Österreichischen Schmerzgesellschaft (ÖSG) arbeiten, rund 10 weitere Schmerzambulanzen befinden sich in Bezirks- und Landeskrankenhäusern [26]. Vor wenigen Jahren waren es aber noch an die 50 Schmerzambulanzen. Schließungen haben weitreichende Konsequenzen für die betroffenen Patienten und das gesamte Gesundheits- und Sozialsystem.

Für eine hohe schmerztherapeutische Versorgungsqualität ist es notwendig, dass genügend Behandlungszentren mit der entsprechenden Ausstattung, personellen sowie zeitlichen Ressourcen zur Verfügung stehen [26, 27]. Sparmaßnahmen, die diese Ressourcen berühren, beeinträchtigen ein optimales Behandlungsangebot und damit die medizinische Versorgung von chronischen Schmerzpatienten. Die Sparmaßnahmen am Gesundheitssektor, wie sie von der Bundesregierung im letzten Sparpaket beschlossen wurden, gefährden somit eine adäquate Schmerzversorgung österreichischer Patienten.

Im niedergelassenen Bereich fehlt eine adäquate multimodale Schmerztherapie völlig bzw. findet nur auf privater Basis statt, da interventionelle oder komplexe schmerztherapeutische Leistungen de facto nicht abgegolten werden.

Zudem gibt es für die Versorgung der laut jüngstem Gesundheitsbericht rund 1,8 Mio. Österreicher mit chronischen Schmerzen keinen Auftrag vonseiten der Politik [15]. Die ambulante Versorgung dieser Patienten ist durch das österreichweite Fehlen einer Bedarfsplanung schmerzmedizinischer Einrichtungen nicht sichergestellt und vorhandene Strukturen - wie spezialisierte Ambulanzen oder Akutschmerzdienste in Krankenhäusern - basieren bisher in erster Linieauf dem persönlichen Engagement Einzelner. Derzeit werden die unterschiedlichen schmerzmedizinischen Einrichtungen in Österreich mit sehr unterschiedlichen Betriebszeiten geführt, sodass aktuellen Schätzungen des Ist- und Sollbedarfs zufolge $74 \%$ der chronischen Schmerzpatienten keine ambulante Schmerztherapie angeboten wird bzw. rund 50 vollzeitbetriebene Schmerzambulanzen fehlen [27].

Unter dem Aspekt, dass sich eine Verbesserung der schmerzmedizinischen Versorgung in Österreich nur erzielen lässt, wenn alle betroffenen Fachgesellschaften und Berufsverbände einheitliche Anforde- rungen an Struktur, Qualität und Qualifikation in der Schmerzmedizin definieren, haben die Österreichische Schmerzgesellschaft (ÖSG), die Österreichische Gesellschaft für Anästhesiologie, Reanimation und Intensivmedizin (ÖGARI), die Allianz Chronischer Schmerz, die Österreichische Gesellschaft für Familienund Allgemeinmedizin (ÖGAM), die Österreichische Gesellschaft für Chirurgie (ÖGC), die Österreichische Palliativgesellschaft (ÖPG), die Österreichische Gesellschaft für Rheumatologie und Rehabilitation (ÖGR), die Österreichische Gesellschaft für Physikalische Medizin und Rehabilitation (ÖGPMR), die Österreichische Gesellschaft für Geriatrie und Gerontologie (ÖGGG), der Berufsverband Österreichischer PsychologInnen (BÖP) sowie der Österreichische Berufsverband für Psychotherapie (ÖBVP) die vorliegende Klassifikation schmerzmedizinischer Einrichtungen in Österreich erarbeitet und konsentiert.

Das nun von Vertretern der Fachgesellschaften formulierte Konzept zur abgestuften intra- und extramuralen Versorgung von Schmerzpatienten (Versorgungspyramide) soll einen ersten Schritt zur Verbesserung darstellen, indem es Leistungs- und Qualitätsstandards unterschiedlicher schmerztherapeutischer Einrichtungen definiert.

Zentrale Forderung der Fachgesellschaften sind festgelegte Struktur- und Qualitätskriterien für schmerzmedizinische Einrichtungen wie z.B. der Nachweis von spezielleren Aus- und Weiterbildungen, die Zusammenarbeit in interdisziplinären Behandlerteams oder die Mindestanzahl an neuen Patienten pro Jahr, je nach Klassifizierung der Einrichtung.

In den interdisziplinären Behandlerteams können Klinische und Gesundheitspsychologen die Versorgung von Schmerzpatienten im Bereich der psychologischen Diagnostik und Behandlung ergänzen. Der psychologische Aspekt steht bei der Berücksichtigung des biopsychosozialen Schmerzmodells zentral. Dieses sieht neben den biologischen Prozessen, die das Syndrombild beeinflussen, auch psychologische und soziale Prozesse involviert. Vor allem für den Beginn, den Schweregrad, die Verschlimmerung und die Aufrechterhaltung von chronischen Schmerzen sind psychologische Faktoren relevant [17].

Ziel der Empfehlungen ist es, verbindliche und überprüfbare Versorgungsstrukturen mit entsprechenden Kriterien zur Qualitätssicherung in der Schmerzmedizin zu etablieren und die Versorgung österreichischer Schmerzpatienten zu verbessern.

\section{Bedarf schmerzmedizinischer Versorgung in Österreich - Status quo}

\section{Hohe Prävalenz von Schmerzzuständen in der Bevölkerung}

Hochrechnungen aus nationalen und internationalen Studien zeigen übereinstimmend eine Prävalenz chronischer Schmerzen (definiert als Schmerzen, die län- 
Abb. 1 Auftreten von Schmerzen in den letzten vier Wochen nach Alter und Geschlecht

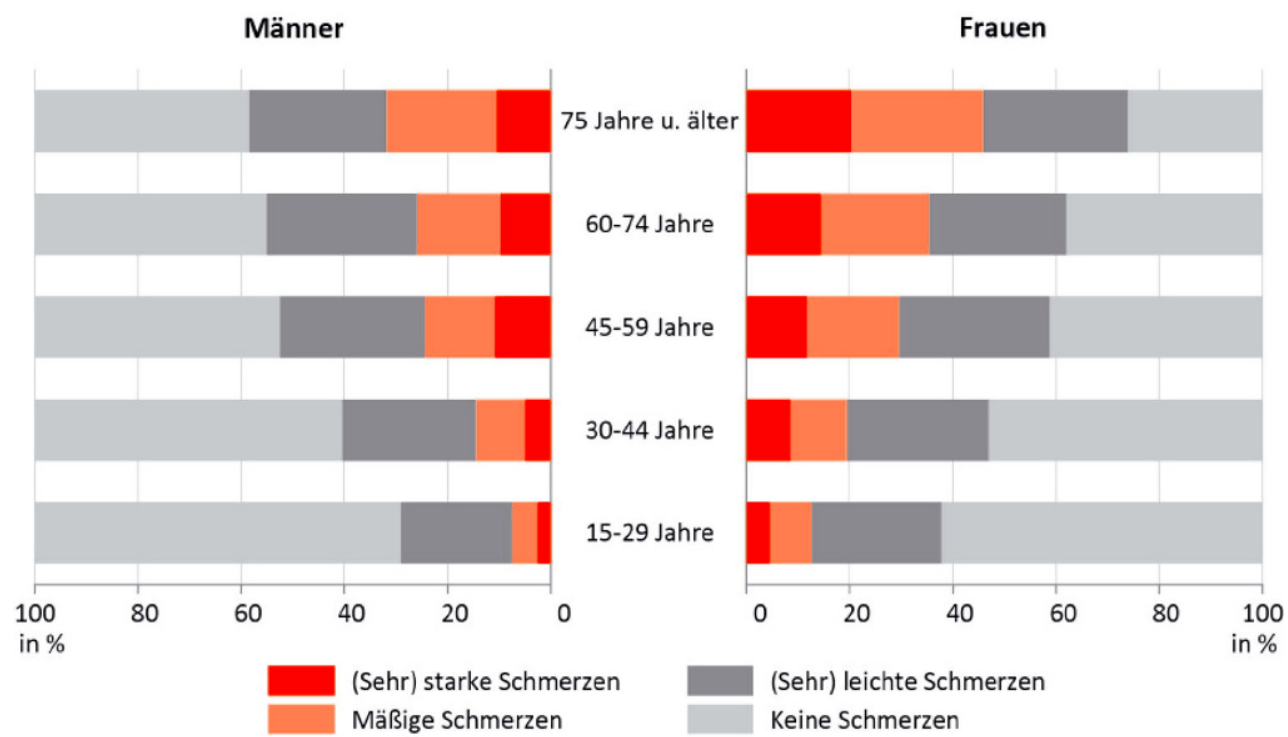

ger als 3-6 Monate andauern) von rund $20 \%[5,22]$. Die IMAS-Umfrage 2009 und der Österreichische Patientenbericht 2009 (beide zitiert in [2, 24, 25]) weichen aber weit von jenen Ergebnissen ab, die im Rahmen des vom Hauptverband der österreichischen Sozialversicherungsträger beauftragten groß angelegten Out come-Forschungsprojekts OF-LÖG (Outcome-Forschung, basierend auf Leistungsdaten des österreichischen Gesundheitswesens) erhoben wurden. Während bei OF-LÖG die Anzahl chronischer Schmerzpatienten, die über 3 Monate verschreibungspflichtige Analgetika einnahmen, mit 4,9\% errechnet wurde, sind laut Gesundheitsbericht aus denselben Jahren (2006/2007) rund ein Viertel der Österreicher von chronischen Schmerzen betroffen [9, 24].

Mögliche Ursachen für die große Diskrepanz könnten in Folgendem bestehen: Eine nicht unerhebliche Anzahl von Personen mit lang anhaltenden Schmerzen behandelt diese mit OTC-Präparaten selbst, ohne einen Arzt aufzusuchen. Viele Patienten brechen die Behandlung mit Analgetika aufgrund von Nebenwirkungen oder schlechter Therapiequalität vorzeitig ab. Diese Patienten wissen oftmals nichts von der Möglichkeit, Schmerzambulanzen aufzusuchen, oder sie werden nicht überwiesen [9].

Dazu kommt, dass ein Teil der Patienten Schmerzen verschweigt oder diese als altersbedingt hinnimmt, wie die Ergebnisse der OSiA-Studie (Optimiertes Schmerzmanagement in Altenpflegeheimen), die in österreichischen Altenheimen durchgeführt wurde, zeigen [23]. Dabei bejahten $68 \%$ der kognitiv leistungsfähigen Heimbewohner, dass Schmerzen im Alter „einfach dazugehören“. Über 40 \% der Befragten gaben an, Schmerz schon einmal verschwiegen $\mathrm{zu}$ haben, wobei als häufigster Grund angegeben wurde, den Pflegenden nicht zur Last fallen zu wollen (64\%). Als weitere Gründe wurden Bedenken wegen Nebenwirkungen $(27 \%)$, bereits kurz zuvor eingenommene
Schmerzmittel (19\%), eine fehlende Bezugspflegekraft (18\%) und Bedenken wegen Medikamentenabhängigkeit $(16 \%)$ genannt [23].

Der letzte Gesundheitsbericht [25] beziffert die Prävalenz chronischer Rückenschmerzen auf Basis einer österreichweiten Erhebung bei 15.771 Personen ab 15 Jahren mit 24,5\%. Hochgerechnet gaben somit 1,8 Mio. Personen an, in den letzten zwölf Monaten unter chronischen Kreuzschmerzen oder einem anderen chronischen Rückenleiden gelitten zu haben (Männer: 23 \%, Frauen: $26 \%$ ). Mit zunehmendem Alter stieg die Häufigkeit dieses gesundheitlichen Problems beträchtlich an: Während bei den Jugendlichen und jungen Erwachsenen jeder zehnte unter Kreuzschmerzen litt, war jeder dritte 60- bis 74-Jährige betroffen. Im höheren Erwachsenenalter waren es sogar $43 \%$ [25]. Beinahe ein Fünftel der Bevölkerung litt unter chronischen Nackenschmerzen oder sonstigen chronischen Beschwerden an der Halswirbelsäule (19\%). Rund $10 \%$ der Männer und $15 \%$ der Frauen gaben an, von Schmerzen sowohl im Rücken- als auch im Nackenbereich betroffen zu sein. $11 \%$ der unter 60-jährigen Frauen, aber nur $4 \%$ der gleichaltrigen Männer gaben an, innerhalb der letzten 12 Monate an chronischen Kopfschmerzen gelitten zu haben. Im Alter von 60 Jahren und mehr lag die Prävalenz bei $7 \%$ der Frauen bzw. $3 \%$ der Männer [30]. An Arthrose leiden im Durchschnitt $8 \%$ der Frauen und $15 \%$ der Männer, bei den 60- bis 74-Jährigen sind es jede dritte Frau und jeder fünfte Mann [25]. Darüber hinaus gaben 3,6 Mio. Personen an, in den letzten vier Wochen Schmerzen gehabt zu haben. Frauen litten häufiger unter Schmerzen als Männer (54\% bzw. $45 \%$ ). Ältere Personen klagten nicht nur öfter über Schmerzen, mit zunehmendem Alter traten auch häufiger starke bzw. sehr starke Schmerzen auf (Abb. 1). 
Diese in der Gesundheitsbefragung 2014 erhobenen Zahlen betreffen Erkrankungen mit dem Leitsymptom Schmerz und nicht nur chronische Schmerzen.

Aktuelle Erhebungen des bundesdeutschen Barmer GEK Ärztereports (Februar 2016) haben ergeben, dass 3,8 Mio. Bundesbürger die Kriterien für chronische Schmerzen mit assoziierten körperlichen, seelischen und sozialen Beeinträchtigungen im Sinne einer „Schmerzkrankheit" erfüllen. Dies würde, für Österreich hochgerechnet, 340.655 Personen betreffen, die der zukünftigen ICD-11-Codierung für chronische Schmerzen entsprechen [28] und nur durch multimodale Therapieansätze eine Chance auf Linderung haben.

Prognosen zufolge wird der Bevölkerungsanteil der über 65-Jährigen bis zum Jahr 2030 um 20 \% zunehmen [21]. Der Bedarf an Schmerzeinrichtungen wird daher mit dem Bevölkerungs- und Altersanstieg deutlich zunehmen.

Vor allem bei Patienten mit demenziellen Erkrankungen sind für die Erkennung und Behandlung von Schmerzen einige Besonderheiten zu berücksichtigen [18]. Schwierigkeiten in der Kommunikation können dazu führen, dass sie seltener über Schmerzen berichten bzw. die Qualität, Lokalisation und Intensität weniger gut beschreiben können als Personen ohne Demenz. Dies kann durch eine adäquate Diagnostik und darauf folgende Behandlung vermieden werden [14].

Auf Basis dieser Ergebnisse bietet sich die Möglichkeit, zielgerichtete Maßnahmen zu ergreifen und damit einen Beitrag zur Verbesserung der öffentlichen Gesundheit und Steigerung der Lebensqualität zu leisten [25].

\section{Schmerzzustände verursachen hohe gesellschaftliche Kosten}

Diese hohe Prävalenz hat nicht nur dramatische Folgen für jeden einzelnen Betroffenen, sondern verursacht auch enorme gesellschaftliche Kosten durch Gesundheitsausgaben, Krankenstandstage, Produktivitätsverlust oder Berufsunfähigkeit [7] bis hin zur Notwendigkeit, Pflege- bzw. Hilfsdienste in Anspruch zu nehmen.

Internationale Studien zeigen einen eklatanten Anstieg der direkten und indirekten Kosten bei Schmerzpatienten in den letzten 20 Jahren [6]. In Österreich sind Erkrankungen des Muskuloskelettalsystems ursächlich für den Verlust von 660.000 Jahresarbeitstagen, die der Hälfte an Fehltagen entsprechen, verantwortlich [3, 29, 10]. Die Kosten dieses Krankheitsbildes betragen etwa 5,5 Mrd. €, jene für Krankenstandstage bei chronischen Rückenschmerzen etwa 400 Mio. $€$. Die direkten Kosten infolge einer Chronifizierung werden mit 1,4-1,8 Mrd. € beziffert.

Dabei konnte gezeigt werden, dass die Rückkehrwahrscheinlichkeit an den Arbeitsplatz mit der Dauer des Arbeitsausfalls korreliert: Beträgt dieser bis zu sechs Monate, liegt die Wahrscheinlichkeit noch bei etwa $50 \%$, bei mehr als einem Jahr sinkt die Wahrscheinlichkeit jedoch auf $20 \%$ und tendiert nach zwei Jahren gegen null Prozent [11].

\section{Langer Leidensweg bis zur korrekten Diagnose und adäquaten Therapie}

Laut dem Pain Proposal Patient Survey (Juli bis September 2010) dauert es im europäischen Schnitt 2,2 Jahre, bis chronische Schmerzpatienten eine adäquate Diagnosestellung erfahren [1]. Chronische Schmerzpatienten frequentieren daher auch häufiger Gesundheitseinrichtungen [4]. Diese Patienten haben bis zu 7 Arztkontakte, $22 \%$ sogar 10 und mehr pro Jahr [1]. In Österreich werden Arztpraxen durchschnittlich 8-mal pro Jahr frequentiert, bis zur Diagnosestellung dauert es durchschnittlich 1,7 Jahre und bis zu einer entsprechenden Behandlung weitere 1,9 Jahre. $18 \%$ der Patienten erfahren keine Diagnosestellung, $23 \%$ keine adäquate Behandlung [2]. Bei $24 \%$ der befragten Personen mit chronischen Schmerzen war die Diagnose erst nach mehr als einem Jahr gestellt worden. $16 \%$ der Patienten erfahren keinerlei Diagnostik, bei $11 \%$ vergehen bis zu einer Diagnosestellung 5-10 Jahre und mehr. Die Diagnose neuropathischer Schmerzen wurde laut einer Studie von Gustorff erst nach Konsultation von durchschnittlich 9 verschiedenen Ärzten gestellt [12].

Die Ergebnisse des Pain Proposal Patient Survey weisen zudem darauf hin, dass sich nicht alle Patienten mit chronischen Schmerzen adäquat behandelt fühlen. $38 \%$ der Befragten waren der Meinung, ihre Schmerzen würden nicht angemessen therapiert, $36 \%$ der Betroffenen haben sogar das Gefühl, dass an der Existenz ihres Schmerzes gezweifelt werde [2].

\section{Beeinträchtigung des Berufslebens}

Die Auswirkungen von chronischen Schmerzen reichen bis in das Arbeitsleben. $61 \%$ der Betroffenen gaben an, dass der chronische Schmerz das Berufsleben unmittelbar beeinträchtige. $21 \%$ der befragten Europäer waren aufgrund ihrer Erkrankung sogar arbeitsunfähig. Im Arbeitsleben stehende Menschen mit chronischen Schmerzen fühlten sich in ihrer Leistungsfähigkeit im Ausmaß von zumindest $28 \%$ eingeschränkt. $29 \%$ gaben an, Angst davor zu haben, den Arbeitsplatz krankheitsbedingt zu verlieren [1]. 26\% der Befragten wurden zudem schon einmal beschuldigt, den chronischen Schmerz als Ausrede zu benutzen, um nicht arbeiten zu müssen [2].

Durchschnittlich stufen Schmerzpatienten ihre Beeinträchtigung beim Verrichten alltäglicher Dinge bei 6,4 (Referenzwerte 1-10), in Österreich bei 5,9 ein [2]. 


\section{Unzureichende Versorgungsstrukturen}

- In Österreich existiert keine flächendeckende Schmerzversorgung. Derzeit gibt es weniger als 40 Schmerzambulanzen in ganz Österreich, die zwischen 3 und 32,5 Wochenstunden geöffnet sind.

- Rechnet man die verfügbaren Ambulanzstunden auf einen 40-Stunden-Betrieb um, so steht für die große Zahl chronisch Schmerzkranker in Österreich ein Äquivalent von nur 17,5 Schmerzambulanzen zur Verfügung.

- Mehrere Einrichtungen mit wenigen Wochenstunden ergeben zusammen nicht eine Schmerzambulanz, die nur annähernd geforderte Kriterien erfüllt [15].

- Tatsache ist, dass in Österreich nur eine einzige multimodale Schmerztagesklinik am Klinikum Klagenfurt besteht, die jedoch vor allem Berufstätigen zur Verfügung steht und zudem zeitlich begrenzt im Rahmen eines Reformpoolprojekts finanziert wird.

- Gemäß einer aktuellen Erhebung wurden in den letzten 5 Jahren 9 Schmerzambulanzen geschlossen; als Schließungsgründe wurden genannt: Personalressourcen (47\%), Zeitressourcen (26\%), Raumressourcen (11\%) und finanzielle Schwierigkeiten (11\%) [27].

- Gründe für das Nichtbetreiben einer Schmerzambulanz: Personalressourcen (36\%), Zeitressourcen (25\%) und eine zu kleine Abteilungsgröße (16\%).

- Eine über 50 \%ige Reduktion des Ambulanzbetriebs während der letzten 3 Jahre wurde in 9 Krankenhäusern berichtet.

- Basierend auf Schätzungen des Ist- und Sollbedarfs, zeigen die Ergebnisse der aktuellen Erhebung, dass in Österreich $74 \%$ der chronischen Schmerzpatienten keine ambulante Schmerztherapie angeboten wird bzw. dass 49,5 vollzeitbetriebene Schmerzambulanzen fehlen.

- Mittlerweile wurden noch weitere Ambulanzen geschlossen, sodass sich die Situation, wenn hier nicht gegengesteuert wird, in den nächsten Jahren noch mehr zu verschärfen droht.

- Die niedergelassenen Ordinationen können die Engpässe, die durch die strukturellen Defizite entstehen, nicht kompensieren: Zum einen ist die Zahl der niedergelassenen Kassenärzte im Verhältnis zur Bevölkerung rückläufig. Zum anderen sind die Diagnostik und Therapie von Patienten mit chronischen Schmerzen zeitlich aufwändig. Dieser Aufwand wird von den Krankenkassen nicht annähernd abgegolten. Schmerztherapie ist de facto im Leistungskatalog der Krankenkassen für Allgemeinmediziner nicht abgebildet [15].

- Zudem bestehen erhebliche Defizite in der Ausbildung zur Schmerztherapie in Österreich. An keiner medizinischen Universität wird Schmerzmedizin nach dem Vorbild Deutschlands als Querschnittsfach unterrichtet $[8,16]$. Wenige Allgemein- mediziner haben das postgraduelle Ärztekammerdiplom „Spezielle Schmerztherapie“ erworben. In Niederösterreich kommen beispielsweise über 22.000 Einwohner auf einen Allgemeinmediziner mit Schmerzdiplom ( $n=72$, Stand November 2014) [15].

- Aufgrund der jüngsten Schließungen und Ressourceneinsparungen und der generell fehlenden Schmerzambulanzen ist zu befürchten, dass hierzulande keine schmerztherapeutische State-of-theart-Versorgung gewährleistet werden kann [27] .

- Auch die Behandlung psychischer Probleme, die mit chronischen Schmerzsyndromen einhergehen können, ist in Österreich nicht flächendeckend gesichert. Da die klinisch-psychologische Behandlung von den Krankenversicherungen nicht abgegolten wird, sondern nur die Kosten für klinisch-psychologische Diagnostik unter bestimmten Umständen übernommen werden [13], ist die benötigte Behandlung für viele betroffene Patienten nicht leistbar.

- Insbesondere im Hinblick auf die häufigen psychischen Komorbiditäten (Depressionen, Angststörungen) bei chronischen Schmerzsyndromen ist es notwendig, die klinisch-psychologische Behandlung in den Leistungskatalog der Krankenkassen aufzunehmen, um den Betroffenen einen multimodalen Behandlungsansatz zu ermöglichen.

\section{Lösung durch strukturelle Verankerung der Schmerztherapie im Gesundheitssystem}

Als Lösungsansatz bietet sich die strukturelle Verankerung der Schmerztherapie im Gesundheitssystem an. In einem ersten Schritt hat der Nationalrat 2015 die Bundesministerin für Gesundheit ersucht, das Bundesinstitut für Qualität im Gesundheitswesen (BIQG) mit der Grundlagenarbeit für Bundesqualitätsstandards zur Verbesserung der Versorgung von Schmerzpatienten in Österreich zu beauftragen. In Unterstützung der Arbeit des BIQG hat die ÖSG unter Einbeziehung anderer Fachgesellschaften ein aktuelles Papier zu Struktur- und Qualitätskriterien für schmerzmedizinische Einrichtungen in Österreich erarbeitet. Das Konzept zur abgestuften Versorgung von Schmerzpatienten soll einen ersten Schritt zur Verbesserung darstellen, indem es Leistungs- und Qualitätsstandards unterschiedlicher schmerztherapeutischer Einrichtungen definiert.

Grundlage sind die hierzulande vorhandenen Strukturen der abgestuften Versorgung sowie bestehende Qualifikationen, Weiterbildungen und Zusatzbezeichnungen. Neben den erstmals beschriebenen schmerzpsychotherapeutischen Einrichtungen lassen sich anhand der Kriterien 3 weitere Typologien definieren: die Ambulanz/Praxis mit gebietsbezogener und schmerztherapeutischer Versorgungsstruktur, die Am- 
bulanz/Praxis für spezielle Schmerztherapie und das interdisziplinäre Schmerzzentrum.

Die interdisziplinäre Besetzung von Schmerzambulanzen im stationären und ambulanten Setting ist schon vielerorts realisiert worden. Allerdings kann diese interdisziplinäre Versorgung außerhalb von Krankenhausstrukturen bisher nicht als gesichert angesehen werden.

\section{Versorgungsrelevanz}

Gemäß der Patientencharta aus dem Jahr 2002 [20] hat jeder Patient Anspruch auf eine medizinische Versorgung. Diagnostik, Behandlung und Pflege haben entsprechend dem jeweiligen Stand der Wissenschaft bzw. nach anerkannten Methoden zu erfolgen. Dabei ist auch der Gesichtspunkt der bestmöglichen Schmerztherapie besonders zu beachten. Ein für alle Patienten erfolgreiches Schmerzmanagement kann nur durch eine enge interprofessionelle Kooperation im Rahmen multimodaler Diagnose- und Therapiekonzepte sowie auf Basis eines biopsychosozialen Schmerzmodells erreicht werden.

Dabei sind eine flächendeckende Bildung regionaler Netzwerke auf allen Versorgungsebenen sowie die Umsetzung integrierter Versorgungsprogramme Voraussetzung dafür, eine unter Koordination des Arztes für Allgemeinmedizin in seiner Funktion als erster Ansprechpartner im Gesundheitssystem stehende abgestufte Versorgung sicherzustellen.

Dafür ist eine adäquate und wohnortnahe schmerzmedizinische Versorgungsstruktur unter Berücksichtigung schmerztherapeutischer Einrichtungen mit definierten Strukturmerkmalen unabdingbar.

Die Bedingung hierfür ist jedoch, dass überprüfbare Kriterien die Strukturen in einem abgestuften Versorgungssystem definieren. Diesem Anspruch folgt die nachfolgende tabellarische Darstellung der verschiedenen schmerztherapeutischen/schmerzmedizinischen Einrichtungen. Sie schafft damit eine Voraussetzung für eine zukünftige Bedarfsplanung schmerzmedizinischer Versorgung.

In der Tab. 2 „Klassifikation schmerzmedizinischer Einrichtungen - Kriterienkatalog" (siehe Anhang) werden Strukturen definiert und beschrieben, in denen die Qualifikation über die allgemeinmedizinische/ fachärztliche Schmerzqualifikation hinausgeht und im Rahmen einer abgestuften Versorgung entweder durch eine besondere Spezialisierung oder durch zunehmend komplexere Strukturen der Einrichtung gekennzeichnet ist (Tab. 1).

Aus diesem Grund schlägt das vorliegende Konsensus-Statement eine Verbesserung der schmerzmedizinischen Basisversorgung durch den Erwerb einer „Spezialisierung Schmerzmedizin“ als Weiterentwicklung des Schmerzdiploms der Österreichischen Ärztekammer vor, mit der die primärärztliche Versorgung verbessert werden kann.
Die nachfolgend genannten Einrichtungen zeichnen sich durch zunehmende Spezialisierung bzw. Komplexität aus. Nach fachgebietsbezogenen Einrichtungen, die nicht ausschließlich schmerzmedizinisch tätig sind, in denen aber auch eine schmerzmedizinische Versorgung vorgehalten wird („Ambulanz/Praxis mit gebietsbezogener und schmerztherapeutischer Versorgungsstruktur“), folgt die Praxis/Ambulanz für spezielle Schmerztherapie, die - immer noch gekoppelt an die fachgebietsbezogene Spezielle Schmerztherapie - ausschließlich oder überwiegend schmerzmedizinisch tätig ist. Darauf folgt das „interdisziplinäre Schmerzzentrum“ mit einem multiprofessionellen und multimodalen Diagnose- und Therapiekonzept. Sonderformen stellen schmerzpsychotherapeutische Einrichtungen dar, die in der Versorgung ebenfalls eine wesentliche Rolle spielen, wie auch interdisziplinäre syndromorientierte Zentren (Kopfschmerz-/Rückenschmerzzentren). Inwieweit multiprofessionelle, multimodale schmerzmedizinische Zentren mit aufwändigen permanenten Kommunikationsprozessen in der Lage sind, eine nennenswerte Anzahl an betroffenen Patienten zu versorgen, bleibt weiteren Untersuchungen vorbehalten.

Die Autoren sind überzeugt, dass mit den hier definierten Struktur- und Qualitätskriterien die schmerzmedizinische Versorgung in Österreich überprüfbar verbessert werden kann und dass diese gleichzeitig Grundlage für Vergütungsregelungen darstellen können.

Interessenkonflikt W. Jaksch, R. Likar, E. Folkes, K. Machold, F. Herbst, K. Pils, P. Stippl, S. Lettner, M. Alfons, R. Crevenna, C. Wiederer, J. Dieber und R. Glehr geben an, dass kein Interessenkonflikt besteht.

Open Access Dieser Artikel wird unter der Creative Commons Namensnennung 4.0 International Lizenz (http:// creativecommons.org/licenses/by/4.0/deed.de) veröffentlicht, welche die Nutzung, Vervielfältigung, Bearbeitung, Verbreitung und Wiedergabe in jeglichem Medium und Format erlaubt, sofern Sie den/die ursprünglichen Autor(en) und die Quelle ordnungsgemäß nennen, einen Link zur Creative Commons Lizenz beifügen und angeben, ob Änderungen vorgenommen wurden.

\section{Anhang}

\section{Qualitätssicherung der schmerzmedizinischen \\ Versorgung in Österreich: Klassifikation schmerzmedizinischer Einrichtungen - Kriterienkatalog}

\section{Präambel}

Unter der Federführung der Österreichischen Schmerzgesellschaft haben verschiedene Fachgesellschaften Struktur- und Qualitätskriterien für schmerzmedizinische Einrichtungen in Österreich definiert, die dazu beitragen sollen, die Versorgungssituation bei Schmerz zu verbessern. 
Tab. 1 Typologien der Einrichtungen

\begin{tabular}{|c|c|c|}
\hline $\mathrm{Nr}$ & Bezeichnung & Kommentar \\
\hline 1 & Interdisziplinäres Schmerzzentrum & $\begin{array}{l}\text { Intramural - Zentrum für interdisziplinäre Schmerzdiagnostik und multimodale } \\
\text { Schmerztherapie }\end{array}$ \\
\hline 2 & Ambulanz/Praxis für spezielle Schmerztherapie & Intra- und extramural \\
\hline 3 & $\begin{array}{l}\text { Ambulanz/Praxis mit gebietsbezogener und schmerztherapeuti- } \\
\text { scher Versorgungsstruktur }\end{array}$ & $\begin{array}{l}\text { Praxis/niedergelassene (extramural) allgemeinmedizinische/fachärztliche Versor- } \\
\text { gung, nicht ausschließlich Schmerztherapie }\end{array}$ \\
\hline 4 & $\begin{array}{l}\text { Schmerzpsychotherapeutische Einrichtung (in Österreich neue } \\
\text { Struktur) }\end{array}$ & $\begin{array}{l}\text { Notwendigkeit muss mit Klinischen Psychologen/Psychotherapeuten abgestimmt } \\
\text { werden }\end{array}$ \\
\hline
\end{tabular}

Tab. 2 Klassifikation schmerztherapeutischer Einrichtungen-Kriterienkatalog

\begin{tabular}{|c|c|c|c|c|c|}
\hline \multirow[t]{2}{*}{$\mathrm{Nr}$} & Bezeichnung & Kommentar & $\begin{array}{l}\text { Interdiszi- } \\
\text { plinäres } \\
\text { Schmerz- } \\
\text { zentrum }\end{array}$ & $\begin{array}{l}\text { Ambulanz/ } \\
\text { Praxis für } \\
\text { spezielle } \\
\text { Schmerzthe- } \\
\text { rapie }\end{array}$ & $\begin{array}{l}\text { Ambulanz/Praxis mit } \\
\text { gebietsbezogener } \\
\text { und schmerzthera- } \\
\text { peutischer Versor- } \\
\text { gungsstruktur }\end{array}$ \\
\hline & & & 1 & 2 & 3 \\
\hline 1 & $\begin{array}{l}\text { Die Einrichtung verfügt über einen eigenständi- } \\
\text { gen Bereich und eine eigene Organisationsstruk- } \\
\text { tur; entsprechend wird eine dafür erforderliche } \\
\text { Infrastruktur vorgehalten } \\
\text { (z. B. Sekretariatswesen, Anmeldung) }\end{array}$ & - & $\mathrm{Ja}$ & Nein & Nein \\
\hline 2 & $\begin{array}{l}\text { Schriftlich niedergelegte SOPs („standard oper- } \\
\text { ating procedures“) für ausgesuchte Krankheits- } \\
\text { bilder, Interventionen, Notfallmanagement sowie } \\
\text { eindeutige Festlegung der Zuständigkeitsberei- } \\
\text { che der verschiedenen Professionen vorhanden }\end{array}$ & SOPs müssen regelmäßig aktualisiert werden & $\mathrm{Ja}$ & $\mathrm{Ja}$ & $\mathrm{Ja}$ \\
\hline 3 & $\begin{array}{l}\text { Dokumentation mit von den Fachgesellschaften } \\
\text { anerkannten Instrumenten zur internen und } \\
\text { externen Qualitätssicherung }\end{array}$ & Qualitätskriterien sind noch auszuarbeiten & $\mathrm{Ja}$ & $\mathrm{Ja}$ & $\mathrm{Ja}$ \\
\hline 4 & $\begin{array}{l}\text { Ärztlicher Leiter mit Zusatzqualifikation „Speziel- } \\
\text { le Schmerztherapie“; } \\
\text { Hinweis, dass verschiedenste Fachrichtungen } \\
\text { infrage kommen (z. B. auch FA für PMR ...) }\end{array}$ & $\begin{array}{l}\text { Zusatzqualifikationen sind noch zu definieren: } \\
\text { Für 1: muss mehr als das Schmerzdiplom } \\
\text { sein } \\
\text { Für 2: Zusatzqualifikation noch zu definieren } \\
\text { Für 3: ÖÄK-Schmerzdiplom }\end{array}$ & $\mathrm{Ja}$ & $\mathrm{Ja}$ & $\mathrm{Ja}$ \\
\hline 5 & $\begin{array}{l}\text { Leiter der Einrichtung weist jährl. } 40 \mathrm{~h} \text { schmerz- } \\
\text { medizinischer Fortbildung und mind. } 10 \text { offene } \\
\text { Schmerzkonferenzen nach; } \\
\text { für Leiter und Mitarbeiter der Einrichtung ist die } \\
\text { schmerzspezifische Fortbildung verpflichtend } \\
\text { zur Beibehaltung der Zusatzqualifikation }\end{array}$ & Ausmaß muss noch definiert werden & $\mathrm{Ja}$ & Nein & Nein \\
\hline 6 & $\begin{array}{l}\text { Verbindliche Kooperationen mit einem Klinischen } \\
\text { Psychologen/Psychotherapeuten }\end{array}$ & $\begin{array}{l}\text { Für } 1+2 \text { : obligat } \\
\text { Für 3: wünschenswert }\end{array}$ & $\mathrm{Ja}$ & $\mathrm{Ja}$ & Nein \\
\hline 7 & $\begin{array}{l}\text { Verbindliche Kooperationen mit einem Physiothe- } \\
\text { rapeuten und/oder Ergotherapeuten }\end{array}$ & $\begin{array}{l}\text { Für } 1+2 \text { : obligat } \\
\text { Für 3: wünschenswert (möglichst regionale } \\
\text { Vernetzung) }\end{array}$ & $\mathrm{Ja}$ & $\mathrm{Ja}$ & Nein \\
\hline 8 & $\begin{array}{l}\text { Behandlerteam aus unterschiedlichen ärztlichen } \\
\text { Fachrichtungen }\end{array}$ & $\begin{array}{l}\text { Obligat: Pflegeassistenzberufe sowie weitere } \\
\text { nichtärztliche Professionen wie Psychothera- } \\
\text { peuten und/oder Ergotherapeuten }\end{array}$ & $\mathrm{Ja}$ & Nein & Nein \\
\hline 9 & $\begin{array}{l}\text { Nichtärztliche Mitarbeiter mit fachspezifischer } \\
\text { Zusatzqualifikation }\end{array}$ & $\begin{array}{l}\text { Mindestens ein Mitarbeiter der jeweils nicht- } \\
\text { ärztlichen Berufsgruppe sollte (sofern vor- } \\
\text { handen oder etabliert) über eine von den hier } \\
\text { beteiligten Fachgesellschaften anerkann- } \\
\text { te schmerzspezifische Zusatzqualifikation } \\
\text { verfügen (z. B. algesiologische Fachassistenz) }\end{array}$ & $\mathrm{Ja}$ & Nein & Nein \\
\hline 10 & $\begin{array}{l}\text { Es existiert eine verbindlich institutionalisierte } \\
\text { Zusammenarbeit zwischen den Professionen } \\
\text { und Disziplinen }\end{array}$ & $\begin{array}{l}\text { Für 1: Tägliche Visiten (bei stationären Pati- } \\
\text { enten) und/oder tägliche interne Fallbespre- } \\
\text { chungen sowie eine mindestens wöchentliche } \\
\text { interdisziplinäre Teambesprechung sind obli- } \\
\text { gat } \\
\text { Für 2: sind Ansprüche des Punktes geringer } \\
\text { zu fassen }\end{array}$ & $\mathrm{Ja}$ & $\mathrm{Ja}$ & Nein \\
\hline 11 & $\begin{array}{l}\text { Die schmerzmedizinische Versorgung wird } \\
\text { an } 5 \text { Wochentagen (ausgenommen Feiertage) } \\
\text { mindestens } 35 \text { h/Woche angeboten }\end{array}$ & $\begin{array}{l}\text { Für 1: obligat } \\
\text { Für 2: Ausmaß muss noch definiert werden }\end{array}$ & $\mathrm{Ja}$ & $\mathrm{Ja}$ & Nein \\
\hline
\end{tabular}


Tab. 2 (Fortsetzung)

\begin{tabular}{|c|c|c|c|c|c|}
\hline \multirow[t]{2}{*}{$\mathrm{Nr}$} & \multirow[t]{2}{*}{ Bezeichnung } & \multirow[t]{2}{*}{ Kommentar } & \multirow{2}{*}{$\begin{array}{l}\text { Interdiszi- } \\
\text { plinäres } \\
\text { Schmerz- } \\
\text { zentrum } \\
1\end{array}$} & \multirow[t]{2}{*}{$\begin{array}{l}\text { Ambulanzl } \\
\text { Praxis für } \\
\text { spezielle } \\
\text { Schmerzthe- } \\
\text { rapie } \\
2\end{array}$} & \multirow{2}{*}{$\begin{array}{l}\text { Ambulanz/Praxis mit } \\
\text { gebietsbezogener } \\
\text { und schmerzthera- } \\
\text { peutischer Versor- } \\
\text { gungsstruktur } \\
3\end{array}$} \\
\hline & & & & & \\
\hline 12 & $\begin{array}{l}\text { Mindestens } 200 \text { Neuvorstellungen pro Jahr sind } \\
\text { an der Einrichtung dokumentiert durchzuführen }\end{array}$ & $\begin{array}{l}\text { Neuvorstellung bedeutet, dass ein Patient } \\
\text { mindestens ein Jahr lang nicht in dieser Ein- } \\
\text { richtung behandelt worden sein darf; jemand, } \\
\text { der von einer anderen schmerzmedizinisch- } \\
\text { en Einrichtung vorbehandelt wurde und an } \\
\text { eine weitere schmerzmedizinische Einrich- } \\
\text { tung überwiesen wird, gilt für letztgenannte } \\
\text { Einrichtung als Neuaufnahme }\end{array}$ & $\mathrm{Ja}$ & Nein & Nein \\
\hline 13 & $\begin{array}{l}\text { Mindestens } 120 \text { Neuvorstellungen pro Jahr sind } \\
\text { an der Einrichtung dokumentiert durchzuführen }\end{array}$ & - & Siehe 12 & $\mathrm{Ja}$ & Nein \\
\hline 14 & $\begin{array}{l}\text { Die Einrichtung verfügt über ein umfassendes } \\
\text { diagnostisches Angebot und Behandlungsspek- } \\
\text { trum für die in der Bevölkerung am häufigsten } \\
\text { vorkommenden Schmerzerkrankungen }\end{array}$ & $\begin{array}{l}\text { Umfassend bedeutet hier, dass die Behand- } \\
\text { lung dem jeweils aktuellen wissenschaftli- } \\
\text { chen Standard entspricht; das diagnostische } \\
\text { Angebot und das Behandlungsspektrum soll- } \\
\text { ten für alle vorhandenen Professionen die von } \\
\text { den zuständigen Fachgesellschaften und/oder } \\
\text { Kammern geforderten Weiterbildungsinhalte } \\
\text { widerspiegeln; diagnostische sowie thera- } \\
\text { peutische Verfahren und die behandelten } \\
\text { Schmerzsyndrome sollten vertreten sein; zu } \\
\text { den relevanten Schmerzerkrankungen, die } \\
\text { diagnostiziert und therapiert werden müssen, } \\
\text { zählen: } \\
\text { (1) Tumorschmerzen } \\
\text { (2) Schmerzen des muskuloskelettalen Sys- } \\
\text { tems } \\
\text { (3) Kopf- und Gesichtsschmerzen } \\
\text { (4) neuropathische Schmerzen } \\
\text { (5) Schmerzen mit psychischen Co-Faktoren } \\
\text { für Auslösung und Chronifizierung }\end{array}$ & $\mathrm{Ja}$ & $\mathrm{Ja}$ & Nein \\
\hline 15 & $\begin{array}{l}\text { Angebote der multimodalen Therapie entspre- } \\
\text { chen den aktuellen konsentierten Konzepten der } \\
\text { Fachgesellschaften }\end{array}$ & $\begin{array}{l}\text { Zusammenarbeit kann auch über regionale } \\
\text { Netzwerke (extramural) erfolgen }\end{array}$ & $\mathrm{Ja}$ & Ja & Ja \\
\hline 16 & $\begin{array}{l}\text { In angemessenem Umfang werden Patienten mit } \\
\text { hohem Chronifizierungsstadium und anderen } \\
\text { Risikokonstellationen (z. B. Medikamentenfehl- } \\
\text { gebrauch, Abhängigkeit, psychische/somatische } \\
\text { Komorbidität) behandelt }\end{array}$ & - & $\mathrm{Ja}$ & $\mathrm{Ja}$ & Nein \\
\hline 17 & $\begin{array}{l}\text { Bei der Erstvorstellung chronischer Schmerz- } \\
\text { patienten müssen die Voraussetzungen für ein } \\
\text { multimodales Eingangsassessment erfüllt wer- } \\
\text { den }\end{array}$ & $\begin{array}{l}\text { Für 1: Das Eingangsassessment } \\
\text { erfolgt obligat durch mind. zwei unterschiedli- } \\
\text { che Fachdisziplinen } \\
\text { (spez. Schmerztherapeut und psychologi- } \\
\text { scher/ärztl. Psychotherapeut/klinischer Psy- } \\
\text { chologe, sowie Physiotherapeut) } \\
\text { Für 2: Auch im regionalen Netzwerk möglich }\end{array}$ & $\mathrm{Ja}$ & Ja & Nein \\
\hline 18 & $\begin{array}{l}\text { Möglichkeit der ambulanten und stationären/ } \\
\text { teilstationären Behandlung vorhanden }\end{array}$ & $\begin{array}{l}\text { Für 2: Bei Ambulanzen wären teilstationäre } \\
\text { Möglichkeiten wünschenswert }\end{array}$ & $\mathrm{Ja}$ & Nein & Nein \\
\hline 19 & $\begin{array}{l}\text { Die Einrichtung führt auch Schmerzforschung } \\
\text { durch }\end{array}$ & $\begin{array}{l}\text { Ausmaß und Art müssen für Österreich noch } \\
\text { definiert werden }\end{array}$ & $\mathrm{Ja}$ & Nein & Nein \\
\hline 20 & $\begin{array}{l}\text { Die Einrichtung ist aktiv an der Ausbildung und/ } \\
\text { oder Weiterbildung und/oder Lehre beteiligt }\end{array}$ & $\begin{array}{l}\text { Für } 3 \text { wäre eine Verpflichtung wünschenswert } \\
\text { - Lehrpraxis }\end{array}$ & $\mathrm{Ja}$ & $\mathrm{Ja}$ & $\mathrm{Ja}$ \\
\hline 21 & $\begin{array}{l}\text { Durchführung von regelmäßigen institutionali- } \\
\text { sierten schmerzmedizinischen Fortbildungsmaß- } \\
\text { nahmen (zwei zertifizierte); jährlich mehrfache } \\
\text { Durchführung von offenen Schmerzkonferenzen }\end{array}$ & $\begin{array}{l}\text { Für 1: obligat } \\
\text { Für } 2 \text { und } 3 \text { : Fortbildung, Workshops, Wis- } \\
\text { sensaustausch; evtl. in einem geringeren } \\
\text { Ausmaß }\end{array}$ & $\mathrm{Ja}$ & $\mathrm{Ja}$ & Ja \\
\hline 22 & $\begin{array}{l}\text { Die volle Weiterbildungsberechtigung (ein Jahr) } \\
\text { für den Erwerb der Zusatzbezeichnung „Speziel- } \\
\text { le Schmerztherapie“ muss vorliegen }\end{array}$ & Zentrum soll ausbilden können & $\mathrm{Ja}$ & Nein & Nein \\
\hline
\end{tabular}


In Österreich existiert keine flächendeckende Versorgung von Patienten mit chronischen Schmerzen. Derzeit gibt es rund 40 Ambulanzen, die offiziell Schmerztherapie in unterschiedlichen Settings anbieten; noch vor wenigen Jahren waren es an die 50. Die Sparmaßnahmen am Gesundheitssektor gefährden somit eine adäquate Schmerzversorgung österreichischer Patienten. Zudem gibt es für die Versorgung der Schätzungen zufolge 1,5 Mio. Österreicher mit chronischen Schmerzen keinen Auftrag vonseiten der Politik. Vorhandene Strukturen wie spezialisierte Ambulanzen oder Akutschmerzdienste in Krankenhäusern basieren bisher in erster Linie auf dem persönlichen Engagement Einzelner.

Das vorliegende Konzept zur abgestuften Versorgung von Schmerzpatienten soll einen ersten Schritt zur Verbesserung darstellen, indem es Leistungs- und Qualitätsstandards unterschiedlicher schmerztherapeutischer Einrichtungen definiert.

\section{Literatur}

1. Baker M, CollettB, FischerA. etal.Improving thecurrentand futuremanagement of chronic pain.AEuropean Consensus Report. 2010.

2. Bernatzky G, Fasching P, Ferraz-Leite H. et al. Verbesserte interdisziplinäreZusammenarbeitin derSchmerztherapie: Forderungen der Ärzteschaft. Positionspapier der ÖSG. 2012.

3. Bevan S, Quadrello T, McGee R. et al. Fit for work? musculoskeletal disorders in the European workforce 2014. www. fitforworkeurope.eu.Zugegriffen:28.11.2016

4. Blyth FM, March LM, Brnabic AJ, Cousins MJ. Chronic pain and frequent use of health care. Pain. 2004;111(1-2):51-8.

5. Breivik H, Collett B, Ventafridda V, Cohen R, Gallacher D. Survey of chronic pain in Europe: prevalence, impact on daily life, and treatment. Eur J Pain. 2006;10(4):287-333.

6. Breivik H, Eisenberg E, O'Brien T, OPENMinds. The individual and societal burden of chronic pain in Europe: the case for strategic prioritisation and action to improve knowledge and availability of appropriate care. BMC Public Health. 2013;13:1229.

7. Czypionka T, Lappöhn S, Pohl A, Röhrling G. Invaliditätspension aufgrund psychischer Erkrankungen. Endbericht. Wien:Institut für Höhere Studien; 2016.

8. Dusch M, Benrath J, Fischer J, et al. Querschnittsbereich 14 Schmerzmedizin - Umsetzung des Kerncurriculums derDeutschen Schmerzgesellschaftim Modellstudiengang MaReCuM. Schmerz. 2013;27:387-94.

9. Endel G, Grögl-Aringer G, Dorda W, et al. Schmerzversorgung in Österreich. SozSicherh. 2013;1:24-36.

10. http://www.fit2work.at/cms/home/attachments/9/5/ 2/CH0080/CMS1426161902414/endbericht_evaluation_ fit2work_end_rev.pdf.Zugegriffen:28.11.2016

11. Frymoyer JW. Predicting disability from low back pain. Clin Orthop RelatRes.1992;. doi:10.1097/00003086-19920600000012.

12. Gustorff B, Dorner T, Likar R, Grisold W, Lawrence K, Schwarz F, Rieder A. Prevalence of self-reported neuropathic pain and impact on quality of life: a pro- spective representative survey. Acta Anaesthesiol Scand. 2008;52(1):132-6.

13. Hauptverband der österreichischen Sozialversicherungsträger. Gesamtvertrag vom 28. November 1994 in der Fassung 1. Zusatzvereinbarung vom 25. November 1996. http://www.wgkk.at/cdscontent/load? contentid=10008. 595920.Zugegriffen:03. März2016.

14. Husebo BS, Ballard C, Sandvik R, et al. Efficacy of treating pain to reduce behavioural disturbances in residents of nursing homes with dementia: Cluster randomised clinical trial. BMJ.2011;343:d4065.

15. Jaksch W. Schmerztherapeutische Versorgungsdefizite in Österreich. Schmerz.2015;29(6):593-4.

16. Kopf A, Dusch M, Alt-Epping B, et al. Das Querschnittsfach „Schmerzmedizin“, Chance für die Allgemeine Schmerzmedizin. Schmerz. 2014;28:405-13.

17. Kröner-Herwig B. Chronischer Schmerz: Psychologische Behandlungsansätze und Stand der Evidenz. VerhaltenstherVerhaltensmed.2014;35(1):57-74.

18. Lukas A, Schuler M, Fischer TW, et al. Pain and dementia. ZGerontolGeriatr. 2012;45:45-9.

19. Österreichische Akademie für Psychologie. Curriculum Psychologische Schmerzbehandlung 2015. https://www. psychologieakademie.at/fortbildung-fuerpsychologinnen/klinische-psychologie/curriculumpsychologische-schmerzbehandlung. Zugegriffen: 28.11.2016

20. Patientencharta 2002. Abrufbar unter: http://www. parlament.gv.at/PAKT/VHG/XXI/I/I_01035/index.shtml

21. Phillips CJ. The cost and burden of chronic pain. Rev Pain. 2009;3(1):2-5.

22. Reid KJ, Harker J, Bala MM, Truyers C, Kellen E, Bekkering GE, Kleijnen J. Epidemiology of chronic non-cancer pain in Europe: narrative review of prevalence, pain treatments and pain impact. Curr Med Res Opin. 2011;27(2):449-62.

23. Schreier MM, Stering U, Pitzer S, Iglseder B, Osterbrink J. Schmerz und Schmerzerfassung in Altenpflegeheimen. Ergebnisse der OSiA-Studie. Schmerz. 2015;29(2):203-10.

24. Statistik Austria. Österreichische Gesundheitsbefragung 2006/2007. Hauptergebnisse und methodische Dokumentation. Wien: StatistikAustria; 2007.

25. Statistik Austria. Österreichische Gesundheitsbefragung 2014. Hauptergebnisse des Austrian Health Interview Survey (ATHIS) und methodische Dokumentation. 2014.

26. Stein KV, Dorner TE, Ilias W, Rieder A. Schmerzpatienten und ihre Erwartungen an die ärztliche Versorgung. Ergebnisse aus dem Österreichischen Patientenbericht. Schmerz. 2010;24:468-73.

27. Szilagyi I-S, Bornemann-Cimenti H, Messerer B, Vittinghoff M, Sandner-Kiesling A. Schmerztherapeutische Versorgung österreichischer Gesundheitszentren. Fragebogenstudie zum Istzustand österreichischer Schmerzambulanzen. Schmerz. 2015;29(6):616-24.

28. Treede RD, Rief W, Barke A, et al. A classification of chronic pain for ICD-11. Pain. 2015;156(6):1003-7.

29. Vavrovsky A, Academy for Value in Health GmbH, Impulsreferat - die Auswirkungen von chronischem Schmerz in Österreich, 4.12.2012

30. Zebenholzer K, Andree C, Lechner A, Broessner G, Lampl C, Luthringshausen G, Wuschitz A, Obmann SM, Berek K, Wöber C. Prevalence, management and burden of episodic and chronic headaches - a cross-sectional multicentre study in eight Austrian headache centres. J Headache Pain. 2015;16:531. 